\title{
Kinetic and Modeling Studies of the
}

\section{Reaction $\mathrm{S}+\mathrm{H}_{2} \mathrm{~S}$}

Yide Gao, ${ }^{1}$ Chenlai (Ryan) Zhou, ${ }^{2}$ Karina Sendt, ${ }^{2}$ A. Goumri, ${ }^{1}$

Brian S. Haynes ${ }^{2}$ and Paul Marshall ${ }^{1}$

${ }^{1}$ Department of Chemistry, University of North Texas, 1155 Union Circle, Denton, Texas

76203-5017, USA

${ }^{2}$ School of Chemical and Biomolecular Engineering, The University of Sydney, Sydney,

NSW 2006, Australia

Word count: 5793 (Method 1)

Main text: 3235

Equations: $\quad 350$

References: 472

Table $1 \quad 73$

$2 \quad 105$

Figure 1: 137 (single-column, incl caption)

Figure 2: $\quad 460$ (two-column, incl caption)

Figure 3: $\quad 430$ (two-column, incl caption)

Figure 4: 531 (two-column, incl caption)

Supplemental material available.

Corresponding Author

Brian S. Haynes

School of Chemical and Biomolecular Engineering

University of Sydney

NSW 2006 AUSTRALIA

Fax $\quad+6129351.3471$

Email: b.haynes@usyd.edu.au 


\section{Abstract}

The reaction of $\mathrm{S}\left({ }^{3} \mathrm{P}_{\mathrm{J}}\right)$ with $\mathrm{H}_{2} \mathrm{~S}$ in Ar bath gas has been characterized by the laser photolysis - resonance fluorescence technique over 300 - $1040 \mathrm{~K}$. The observed secondorder rate constants are found to be pressure-dependent below $700 \mathrm{~K}$. The reaction has been modeled accurately as a combination of direct abstraction on the triplet surface

$$
\mathrm{S}\left({ }^{3} \mathrm{P}_{\mathrm{J}}\right)+\mathrm{H}_{2} \mathrm{~S} \rightarrow 2 \mathrm{SH}
$$

together with multi-well reactions on the singlet surface via intersystem crossing

$$
\begin{aligned}
& \mathrm{S}\left({ }^{3} \mathrm{P}_{\mathrm{J}}\right)+\mathrm{H}_{2} \mathrm{~S} \rightarrow \mathrm{H}_{2} \mathrm{SS}^{*} \rightarrow \mathrm{HSSH}^{*}+\mathrm{M} \rightarrow \mathrm{HSSH}+\mathrm{M} \\
& \mathrm{S}\left({ }^{3} \mathrm{P}_{\mathrm{J}}\right)+\mathrm{H}_{2} \mathrm{~S} \rightarrow \mathrm{H}_{2} \mathrm{SS}^{*} \rightarrow \mathrm{HSSH}^{*} \rightarrow 2 \mathrm{SH}
\end{aligned}
$$

Since the intersystem crossing has a low energy barrier ( $3 \mathrm{~kJ} \mathrm{~mol}^{-1}$ ), the spin-forbidden channels dominate at low temperature, with the overall rate and product distribution being dependent on pressure. Above $800 \mathrm{~K}$, the abstraction channel on the triplet surface becomes important and is dominant above $1000 \mathrm{~K}$. 


\section{Introduction}

Thiyl (SH) and atomic sulfur are important radical intermediates in the combustion chemistry of sulfur [1]. They may be involved in coupling sulfur and nitrogen chemistry in flames, where the mechanistic details of how fuel sulfur influences the formation of $\mathrm{NO}_{\mathrm{x}}$ during combustion are unknown [2,3]. They are also important in the chemistry of $\mathrm{H}_{2} \mathrm{~S}$ pyrolysis, gasification and the homogeneous Claus reaction [4-6].

The reaction

$$
\mathrm{S}\left({ }^{3} \mathrm{P}_{\mathrm{J}}\right)+\mathrm{H}_{2} \mathrm{~S} \rightarrow \text { products }
$$

may couple sulfur species at elevated temperatures. The direct abstraction reaction on the triplet surface leads to formation of two SH radicals (2) with a computed barrier of $45 \mathrm{~kJ}$ $\mathrm{mol}^{-1}$ [7] about $19 \mathrm{~kJ} \mathrm{~mol}^{-1}$ above the endothermicity of the reaction

$$
\mathrm{S}\left({ }^{3} \mathrm{P}_{\mathrm{J}}\right)+\mathrm{H}_{2} \mathrm{~S} \rightarrow 2 \mathrm{SH} \quad \Delta \mathrm{H}_{0 \mathrm{~K}}=26 \mathrm{~kJ} \mathrm{~mol}^{-1}
$$

Shiina et al. [8] found reaction (1) to proceed with a barrier close to the endothermicity of (2) and suggested that formation of $\mathrm{SH}+\mathrm{SH}$ occurs via a triplet-singlet crossing and insertion. This possibility was confirmed in high-level theoretical work [7] which showed that the low singlet barrier relative to the direct abstraction reaction (1) on the triplet surface would make the surface crossing route especially important at low temperatures. Zhou et al. [9] subsequently carried out a detailed study of different channels on the multi-well triplet and singlet $\mathrm{H}_{2} / \mathrm{S}_{2}$ surfaces. As shown in Fig. 1, which represents the relevant parts of the PES, the intersystem crossing (ISC) is predicted to lie $14 \mathrm{~kJ} \mathrm{~mol}^{-1}$ above the reactants $\mathrm{H}_{2} \mathrm{~S}+\mathrm{S}$. An excited triplet complex $\mathrm{H}_{2} \mathrm{SS}^{*}$ can cross to the singlet 
surface where it is able to rearrange to the excited isomer $\mathrm{HSSH}^{*}$, stabilize as $\mathrm{H}_{2} \mathrm{SS}$ to a minor extent, or recross to form reactants. Subsequently, the excited $\mathrm{HSSH}^{*}$ may dissociate to $\mathrm{SH}+\mathrm{SH}$, be collisionally stabilized as $\mathrm{HSSH}$ which is the most stable molecule in the $\mathrm{H}_{2} / \mathrm{S}_{2}$ system, or return to $\mathrm{H}_{2} \mathrm{SS}^{*}$. The net apparent reactions are then

$$
\begin{aligned}
& \mathrm{S}\left({ }^{3} \mathrm{P}_{\mathrm{J}}\right)+\mathrm{H}_{2} \mathrm{~S} \rightarrow \mathrm{H}_{2} \mathrm{SS}^{*} \rightarrow \mathrm{HSSH}^{*}+\mathrm{M} \rightarrow \mathrm{HSSH}+\mathrm{M} \\
& \mathrm{S}\left({ }^{3} \mathrm{P}_{\mathrm{J}}\right)+\mathrm{H}_{2} \mathrm{~S} \rightarrow \mathrm{H}_{2} \mathrm{SS}^{*} \rightarrow \mathrm{HSSH}^{*} \rightarrow 2 \mathrm{SH}
\end{aligned}
$$

While this new work also showed that the dissociation of the excited singlet species to HSS $+\mathrm{H}$ becomes significant ( $>5 \%$ ) relative to dissociation to $\mathrm{SH}+\mathrm{SH}$ at temperatures above $1500 \mathrm{~K}$, the essential features of the PES for reaction of $\mathrm{H}_{2} \mathrm{~S}+\mathrm{S}$ are as described in Fig. 1. However, there remain a number of areas of uncertainty in the determination of the rates on the singlet surface, including the collisional stabilisation of the excited species on the singlet surface and the estimation of the surface crossing frequency. Since the insertion channel is expected to play a greater role at lower temperatures, we have investigated the system through kinetic measurements of the overall rate of $\mathrm{H}_{2} \mathrm{~S}+\mathrm{S}$ reaction at temperatures from 296 to $1040 \mathrm{~K}$ and over a range of pressures from 13 to 660 mbar. Modelling of the multiple-well system then reveals more details about the process and produces accurate chemical kinetic rate parameters. 


\section{Experimental Method and Results}

Hydrogen sulfide (“99.5\%” grade from MG Industries) was purified first by trapping at $77 \mathrm{~K}$ (liquid $\mathrm{N}_{2}$ ) and degassing, which was repeated for several “freeze-pump-thaw” cycles. The $\mathrm{H}_{2} \mathrm{~S}$ was then distilled repeatedly from $210 \mathrm{~K}$ (chloroform slush) and trapped at 77 K. Carbon disulfide (Aldrich, “99.9+\%”) was trapped and degassed at $196 \mathrm{~K}$ (acetone slush). Argon was used directly from the cylinder (99.995\%, Air Liquide and Big Three). Gas mixtures were prepared manometrically in glass bulbs.

The laser photolysis apparatus and general operating procedures have been described elsewhere [10-13]. Briefly, mixtures of $\mathrm{H}_{2} \mathrm{~S}$ and $\mathrm{CS}_{2}$ diluted in Ar bath gas flowed slowly through a heated, stainless steel reaction cell. Actinic radiation entered through one side arm and probe radiation from a resonance lamp through a second side arm, and fluorescence was observed through a third mutually perpendicular side arm. The temperature in the reaction zone, defined by the intersection of the three side arms, was measured with a retractable, sheathed, unshielded thermocouple corrected for radiation errors [14].

Atomic sulfur was generated from pulsed UV laser photolysis of $\mathrm{CS}_{2}$ precursor at 193 $\mathrm{nm}$. The pulse repetition rate was $1-2 \mathrm{~Hz}$ to allow fresh gas mixtures to enter the reaction zone between pulses. The laser beam had a cross section of about $0.6 \mathrm{~cm}^{2}$. At $193 \mathrm{~nm}$ the absorption cross section of $\mathrm{CS}_{2}$ is $1.3 \times 10^{-16} \mathrm{~cm}^{2}$ molecule ${ }^{-1}$ [15]. For estimation of initial atom concentrations we assume a unit quantum yield for dissociation, although this information is not needed for the kinetic analysis described below. The ratio of $\mathrm{S}\left({ }^{3} \mathrm{P}\right)$ to 
$\mathrm{S}\left({ }^{1} \mathrm{D}\right)$ in the products has been reported as $1.6 \pm 0.3[16]$. The $S\left({ }^{1} \mathrm{D}\right)$ atoms are expected to be quenched rapidly in our system by collision with the Ar bath gas: the quenching rate constant is $1.4 \times 10^{-11} \mathrm{~cm}^{3}$ atom $\mathrm{s}^{-1}$ [17], and with [Ar] at over $10^{17}$ atom $\mathrm{cm}^{-3}$, the collisional lifetime of $S\left({ }^{1} \mathrm{D}\right)$ is less than $1 \mu \mathrm{s}$ which is orders of magnitude shorter than the time scale of the kinetic measurements. To estimate the extent of $\mathrm{H}_{2} \mathrm{~S}$ photolysis at $193 \mathrm{~nm}$, an absorption cross section of $6.50 \times 10^{-18} \mathrm{~cm}^{2}$ molecule ${ }^{-1}$ and a quantum yield of 0.23 were employed [18].

Inside the resonance lamp, around 0.4 mbar of a mixture of $0.1 \%$ of $\mathrm{H}_{2} \mathrm{~S}$ in Ar flowed through a microwave discharge to generate probe radiation at $\lambda \approx 181 \mathrm{~nm}$ (sulfur $(4 \mathrm{~s}){ }^{3} \mathrm{~S}$ $\left.\rightarrow(3 p){ }^{3} \mathrm{P}\right)$ [19]. This radiation was focused through Suprasil quartz optics into the center of the reactor and excited resonance fluorescence from S-atoms in the reaction zone. The time-resolved fluorescence was detected with a solar-blind photomultiplier tube operated in the photon counting mode, with a Suprasil window. This arrangement avoided interference from any $S_{2}$ and/or $S_{3}$ emission in the near UV. Signals following up to 1000 photolysis pulses were accumulated in a multi-channel scaler.

After their generation, S-atoms decay mainly through reaction with $\mathrm{H}_{2} \mathrm{~S}$ and with $\mathrm{CS}_{2}$. The decrease in atomic sulfur concentration may be described by

$$
\mathrm{d}[\mathrm{S}] / \mathrm{dt}=-\left(k_{1}[\mathrm{~S}]\left[\mathrm{H}_{2} \mathrm{~S}\right]+k^{\prime}[\mathrm{S}]\right)=-k_{p s 1}[\mathrm{~S}]
$$

where $k^{\prime}$ accounts for loss of S by reaction with $\mathrm{CS}_{2}$ (whose concentration is held constant in each series of decays to determine $k_{1}$ ), diffusion and any secondary chemistry. Because the concentration of $\mathrm{S}$ is much smaller than that of $\mathrm{H}_{2} \mathrm{~S}$, pseudo-first- 
order kinetics are expected. $k_{p s 1}$ is determined from non-linear least squares fitting of the fluorescence signals to exponential decays. An example is shown as the inset on Fig. 2. A linear plot of $k_{p s 1}$ vs. [ $\mathrm{H}_{2} \mathrm{~S}$ ] at constant precursor concentration, such as Fig. 2, has a slope equal to the effective second order rate constant $k_{1}$. Experiments were carried out to determine the dependence of $k_{1}$ on temperature $\mathrm{T}$ and [Ar], and to investigate the influence of experimental parameters such as the laser pulse energy $F$ and the average gas residence time inside the heated cell before photolysis $\tau_{\text {res, }}$, as well as the initial radical concentrations.

Table 1S in the Supplemental Material summarizes 72 measurements made at 9 temperatures from 300 to $1040 \mathrm{~K}$. At each T and P, $k_{p s 1}$ was determined as function of $\left[\mathrm{H}_{2} \mathrm{~S}\right]$ at five values from zero to $\left[\mathrm{H}_{2} \mathrm{~S}\right]_{\max }$ to yield $k_{1}$. There is no systematic deviation of $k_{1}$ with the experimental parameters $\mathrm{F},[\mathrm{SH}]_{0},[\mathrm{~S}]_{0}$ and $\tau_{\text {res, }}$, which demonstrates that thermal decomposition of the $\mathrm{H}_{2} \mathrm{~S}$ was unimportant and that reaction 1 has been successfully isolated from secondary chemistry. This latter point is also addressed via kinetic modeling described in the Discussion. Plots of $k_{1}$ vs. Ar density (Fig. 3) indicate a pressure dependence for the total rate constant $k_{1}$ below $700 \mathrm{~K}$, switching to pressureindependent values at higher temperatures (see Table 1S). This behavior is rationalized quantitatively in the following sections. 


\section{Computational Approach}

The potential energy surface (PES) of the reaction $\mathrm{H}_{2} \mathrm{~S}+\mathrm{S}$ was characterized at the MRCI level and the kinetic study of pressure-dependent channels was performed using the Multiwell program suite [20].

While the thermochemistry of $\mathrm{H}_{2} \mathrm{~S}+\mathrm{S}, \mathrm{HSSH}$ and $\mathrm{SH}+\mathrm{SH}$ used in previous work [9] is in good agreement with the established literature values, the enthalpy of formation of $\mathrm{H}_{2} \mathrm{SS}$ determined at the $\operatorname{CSD}(\mathrm{T})$ level with extrapolation to a complete basis set was found to be $6.9 \mathrm{~kJ} \mathrm{~mol}^{-1}$ (extrapolated to $0 \mathrm{~K}$ ) lower than our MRCI value [21]. The approximation of the CBS limit and the additional correction of scalar relativistic effects estimated at the DKCCSD(T)/cc-PVQZ_DK level of theory make this new value preferable to the MRCI energy, especially as there is scant evidence of multi-configurational character in the $\mathrm{H}_{2} \mathrm{SS}$ molecule which shows a Q1 diagnostic factor of 0.016 in the QCISD(T) contribution to G3 and a largest CI coefficient of 0.976 in our CASSCF calculations. While there remains a need for experimental verification [21], we have adopted the value, $\Delta H_{\mathrm{f}}{ }^{0} 298\left(\mathrm{H}_{2} \mathrm{SS}\right)=123 \pm 4 \mathrm{~kJ} \mathrm{~mol}^{-1}$ in Fig. 1 and in the Multiwell simulations.

Collisional energy transfer in Ar bath gas has been modeled using Lennard-Jones collision parameters for $\operatorname{Ar}[22]$ with average energies $<\Delta \mathrm{E}>$ transferred per collision of excited $\mathrm{HSSH}^{*}$ and $\mathrm{H}_{2} \mathrm{SS}^{*}$ taken from experimental values for $\mathrm{SO}_{2}$ in $\mathrm{Ar}$ over the temperature range $300-700 \mathrm{~K}$ [23]. Values of $<\Delta \mathrm{E}>$ were converted to average energies $<\Delta \mathrm{E}_{\text {down }}>$ for down transitions via the relation [22] 


$$
-<\Delta \mathrm{E}>\approx \frac{<\Delta \mathrm{E}_{\mathrm{down}}>^{2}}{<\Delta \mathrm{E}_{\mathrm{down}}>+\mathrm{kT}}
$$

and correlated against temperature and system energy, yielding, for $\mathrm{E}<33500 \mathrm{~cm}^{-1}$ (i.e. $400 \mathrm{~kJ} \mathrm{~mol}^{-1}$ )

$$
<\Delta \mathrm{E}_{\text {down }}>\left(\mathrm{cm}^{-1}\right)=2.23 \times 10^{-3}\left(\frac{\mathrm{T}(\mathrm{K})}{300}\right)^{1.4} \mathrm{E}\left(\mathrm{cm}^{-1}\right)
$$

Equation (2) captures the experimental data in the region of interest to within $5 \%$ and has been used in the exponential down model for the collision process in the Multiwell simulations. At the crossing energy in the present system, and for temperatures from 300 to $1000 \mathrm{~K}$, equation (2) predicts $<\Delta \mathrm{E}_{\text {down }}>$ to go from 45 to $240 \mathrm{~cm}^{-1}$, compared with the value of $230 \mathrm{~cm}^{-1}$ we used in earlier work for $\mathrm{N}_{2}$ [9].

Detailed description of the computational methods has been presented in previous work [9].

\section{Discussion}

The H/S system is highly reactive [4]. We therefore assessed the impact of other reactions on the experimental using an updated H/S mechanism [24] and confirmed that the effect was minor: the experimentally-observed rates of disappearance of S were at most $20 \%$ greater than the predicted overall rate of reaction (1), the main enhancement being reaction of $\mathrm{S}$ with $\mathrm{SH}$ that was formed in the flash photolysis. We therefore take the raw experimental data as the rate of the target reaction without modification. 
We also considered experimental data from Shiina et al. [8] at higher temperatures (1050$1660 \mathrm{~K})$. In this case, the formation of SH in the flash photolysis was assumed to be negligible, as stated by the authors [8], but the contribution of secondary reactions is now predicted to be significant, with the observed rates of S-atom consumption being as much as 1.7 times greater than the rate of $\mathrm{H}_{2} \mathrm{~S}+\mathrm{S}$.

The Multiwell predictions are very sensitive to the presumed ISC barrier height. The calculated value of $E_{\text {ISC }}=14 \mathrm{~kJ} \mathrm{~mol}^{-1}$ [7] proved to be too high for the low-temperature results to be modeled - the predicted rate of reaction was too low by an order of magnitude and its apparent activation energy under the higher-pressure conditions was too high. We therefore adjusted the ISC barrier to $3 \mathrm{~kJ} \mathrm{~mol}^{-1}$ in order to capture the highand low-pressure experimental trends. Figure 4 compares the predicted and measured rate constants with respect to $\mathrm{S}$ atom disappearance as a function of temperature at high (550 mbar) and low (13 mbar) pressures. The simulations in Fig. 4 use an intersystem crossing efficiency (based on the $\mathrm{H}_{2} \mathrm{~S}+\mathrm{S}$ collision frequency) of $20 \%$ and $<\Delta \mathrm{E}_{\text {down }}>$ as given by equation (2) - these parameters, with $\mathrm{E}_{\mathrm{ISC}}=3 \mathrm{~kJ} \mathrm{~mol}^{-1}$, constitute the base case for the modeling.

The reduction of the ISC barrier by $11 \mathrm{~kJ} \mathrm{~mol}^{-1}$ is at the upper limit of errors normally expected in the MRCI calculations and we therefore investigated the crossing energy using larger basis sets. At the optimized MRCI/aug-cc-pV(T+d)Z geometry reported earlier [7], the S-S bond length $\mathrm{H}_{2} \mathrm{SS}^{*}$ was found to be $2.658 \AA$, and the barrier $20 \mathrm{~kJ}$ $\mathrm{mol}^{-1}$. Single point calculations using the aug-cc-pV(Q+d)Z basis set reduced this barrier 
to $14 \mathrm{~kJ} \mathrm{~mol}^{-1}$ [7]. Reoptimizing the crossing geometry with the aug-cc-p V(Q+d)Z basis set increased the S-S bond length slightly to $2.670 \AA$ and further reduced the barrier to $12 \mathrm{~kJ} \mathrm{~mol}^{-1}$. Subsequent single point calculations with the aug-cc-pV(5+d)Z basis set placed the barrier at $11 \mathrm{~kJ} \mathrm{~mol}^{-1}$ or $8 \mathrm{~kJ} \mathrm{~mol}^{-1}$ greater than the fitted value. We note that the geometry at the crossing point resembles $\mathrm{H}_{2} \mathrm{SS}$ and suggest that the source of the inaccuracy in the prediction of the $\mathrm{H}_{2} \mathrm{SS}$ energy at the MRCI level discussed above may also affect the energy of $\mathrm{H}_{2} \mathrm{SS}^{*}$ which would resolve the discrepancy in the crossing energy. However, it should also be noted, the calculations are only an approximation of the true crossing condition, matching the point at which the optimised triplet geometry has the same energy on the singlet surface [7] but ignoring dynamical effects - in a dynamical study of the $\mathrm{S}+\mathrm{H}_{2}$ reaction, Maiti et al. [25] noted the sensitivity of their results to details of coupled surface dynamics near the point of crossing of the singlet and triplet states. We therefore conclude that our empirical adjustment of the barrier is reasonable.

In Figure 4, the experimental data at $\mathrm{p}=13$ and $\mathrm{p}=550$ mbar show a strong pressure dependence at low temperatures that diminishes with increasing temperature and vanishes above $660 \mathrm{~K}$, and these results are closely matched by the sum of the rates on the triplet and singlet surfaces (shown by a dashed line). Note that the experiments were not carried out at pressures up to 550 mbar at T> $660 \mathrm{~K}$ but the complete absence of pressure dependence was confirmed. 
Also shown in Fig. 4 are the calculated rates for the reaction on the singlet surface as $\mathrm{p} \rightarrow \infty$ (all product $\mathrm{HSSH}$ ) and $\mathrm{p} \rightarrow 0$ (all product $\mathrm{SH}+\mathrm{SH}$ ) - these converge above $800 \mathrm{~K}$ where the rate, but not the product distribution, is predicted to become independent of pressure. The contributions of reaction on the singlet surface to the overall reaction at 13 and at 550 mbar are shown as dotted lines - these are hard to discern from the overall rate at lower temperatures, where the rate on the triplet surface is negligible, but differences appear at higher temperatures as the reaction occurs increasingly on the triplet surface. Table 1 reports the kinetic parameters for the various processes as pseudo-elementary reactions, in a form suitable for use in chemical kinetic modeling. Here it should be noted that the expression for the dissociation channel (3b) has been evaluated on the basis of the present simulations and care should be taken in applying this above $\sim 1$ bar.

The data of Shiina et al. [8] align well with the present work, especially after correction for secondary reactions in their experiments, and are also accurately modeled. It is apparent that most of the reaction in those experiments is in fact via the triplet channel. The low activation energy observed in the original data is due in part to parallel reaction on the singlet channel, as proposed, but it appears that secondary reactions may also have played a role in reducing the experimental temperature sensitivity.

Figure 3 shows that the prediction of the detailed pressure dependence of the rate constants under conditions where most of the reaction is on the singlet surface. The reaction shows typical fall-off behavior at low temperatures, but this is increasingly 
compressed as the temperature increases and the dissociation channel (R3b) becomes competitive with the high-pressure limit of the stabilization pathway.

The Multiwell modelling provides a detailed description of the reaction processes. At low temperatures, the kinetics are dominated by channel R3, forming singlet products. While $\mathrm{SH}+\mathrm{SH}$ is the major product at low pressures, the stabilization of HSSH becomes important as the pressure increases. This effect is responsible for the increase in the overall reaction rate shown in Fig. 4 in going from low to high pressures since a significantly greater proportion of the excited adduct $\mathrm{H}_{2} \mathrm{SS} *$ reverts to reactants at low pressures (94\% at 13 mbar and $296 \mathrm{~K}$ ) versus high (50\% at $550 \mathrm{mbar}$ ). As the temperature increases, the singlet dissociation channel to $\mathrm{SH}+\mathrm{SH}$ is relatively more favored, having a further activation barrier of $23 \mathrm{~kJ} \mathrm{~mol}^{-1}$, and the pressure dependence weakens. The proportion of HSSH in the products at 550 mbar therefore falls from $97 \%$ at $296 \mathrm{~K}$ to $37 \%$ at $560 \mathrm{~K}$ and is negligible above about $700 \mathrm{~K}$. At temperatures in excess of $1000 \mathrm{~K}$, the triplet bimolecular abstraction channel (2) becomes dominant.

\section{Sensitivity analysis}

We have investigated the sensitivity of the Multiwell simulations to the most significant parameters in the model, namely the ISC barrier height, the crossing efficiency and $<\Delta \mathrm{E}_{\text {down }}>$. For the barrier, a perturbation of $\pm 3 \mathrm{~kJ} \mathrm{~mol}^{-1}$ was applied, while the sensitivity of crossing efficiency and $<\Delta \mathrm{E}_{\text {down }}>$ were studied by scaling up and down by a factor of 1.5 relative to the base case. In Table 2, we present the results of these runs at $296 \mathrm{~K}-$ the magnitude of changes in the rates were similar for upward and downward 
perturbation and we report only the average. Similar results were obtained at $490 \mathrm{~K}$ but sensitivity to parameters for reaction on the singlet surface rapidly falls rapidly after that.

As discussed above, the formation of HSSH is very sensitive to the ISC barrier, especially at low temperatures. For $\Delta \mathrm{E}_{\mathrm{ISC}}= \pm 3 \mathrm{~kJ} \mathrm{~mol}^{-1}$, the rate of formation of $\mathrm{HSSH}$ changes by factors of 3 and 2 at $296 \mathrm{~K}$ and $490 \mathrm{~K}$ respectively, but there is no significant change in the rate to $\mathrm{SH}+\mathrm{SH}$ because the overall barrier to this endothermic channel is unchanged. Variation in $<\Delta \mathrm{E}_{\text {down }}>$ also directly impacts on HSSH formation, especially at low pressures - thus a multiplicative variation of 1.5 increases the rate by 1.8 times at 13 mbar and by 1.4 times at 550 mbar, relatively independently of temperature and without significant effect on the rate of formation of $\mathrm{SH}+\mathrm{SH}$. Clearly, the values of $\Delta \mathrm{E}_{\text {ISC }}$ and $<\Delta \mathrm{E}_{\text {down }}>$ required to model the data show some interaction. However, without invoking a different temperature dependence for $<\Delta \mathrm{E}_{\text {down }}>$ than is given by equation (2), we still found the value of $\mathrm{E}_{\text {ISC }}=3 \mathrm{~kJ} \mathrm{~mol}^{-1}$ to provide the best representation of the temperature-dependence of low-temperature, high-pressure data.

Variations in the ISC efficiency $\eta_{\text {IsC }}$ affect the rates of all processes, not quite proportionally because variation in the rate of rate of crossing also affects the return of excited ${ }^{1} \mathrm{H}_{2} \mathrm{SS}^{*}$ to reactants.

\section{Conclusions}

The reaction of $\mathrm{S}\left({ }^{3} \mathrm{P}_{\mathrm{J}}\right)$ with $\mathrm{H}_{2} \mathrm{~S}$ in Ar bath gas, characterized by the laser photolysis resonance fluorescence technique over 300 - $1040 \mathrm{~K}$, shows a complex temperature- 
dependence and is also pressure-dependent below $700 \mathrm{~K}$. The present experimental results and literature values at higher temperatures are rationalized through taking into account both the direct abstraction on the triplet surface and the multi-well reactions on the singlet surface via intersystem crossing. Excited species on the singlet surface $\left(\mathrm{H}_{2} \mathrm{SS}^{*}\right.$ and $\mathrm{HSSH}^{*}$ ) may stabilize, chiefly as $\mathrm{HSSH}$, or dissociate to $\mathrm{SH}+\mathrm{SH}$.

The intersystem crossing has a low energy barrier $\left(3 \mathrm{~kJ} \mathrm{~mol}^{-1}\right)$, allowing the spinforbidden channels dominate at low temperature, with the overall rate and product distribution being dependent on pressure. Above $800 \mathrm{~K}$, the abstraction channel on the triplet surface becomes important and is dominant above $1000 \mathrm{~K}$.

\section{Acknowledgments}

The UNT work was supported by the National Science Foundation (Grant CBET0756144), the Robert A. Welch Foundation (Grant B-1174) and the UNT Faculty Research Fund. The work at University of Sydney was supported in part by the Australian Research Council and carried out on the NCI National Facility in Canberra, Australia. Chenlai (Ryan) Zhou thanks the School of Chemical and Biomolecular Engineering of the University of Sydney for the award of the FH Loxton Postgraduate Studentship. 


\section{References}

(1) A.J. Hynes, P.H. Wine, in W.C. Gardiner, Jr. (Ed.), Gas-Phase Combustion Chemistry. Springer-Verlag, New York, 2000, Ch. 3.

(2) A.T. Chen, P.C. Malte, M.M. Thornton, Proc. Combust. Inst. 20 (1984) 769.

(3) W. Nimmo, E. Hampartsoumian, K.J. Hughes, A.S. Tomlin, Proc. Comb. Inst. 27 (1998) 1419.

(4) K. Sendt, M. Jazbec, B.S. Haynes, Proc. Combust. Inst. 29 (2002) 2439.

(5) K. Sendt, M. Jazbec, B.S. Haynes, Fuel 83 (2004) 2133.

(6) I.A. Gargurevich, Ind. Eng. Chem. Res. 44 (2005) 7706.

(7) C. Zhou, K. Sendt, B.S. Haynes, J. Phys. Chem. A 112 (2008) 3239.

(8) H. Shiina, M. Oya, K. Yamashita, A. Miyoshi, H. Matsui, J. Phys. Chem. 100 (1996) 2136.

(9) C. Zhou, K. Sendt, B.S. Haynes, J. Phys. Chem. A 113 (2009) 8299.

(10) Y. Shi, P. Marshall, J. Phys. Chem. 95 (1991) 1654.

(11) L. Ding, P. Marshall, J. Phys. Chem. 96 (1992) 2197.

(12) L. Ding, P. Marshall, J. Chem. Phys. 98 (1993) 8545.

(13) A. Goumri, D.D. Shao, P. Marshall, J. Chem. Phys. 121 (2004) 9999.

(14) L. Ding, P. Marshall, J. Chem. Soc. Faraday Trans. 89 (1993) 419.

(15) W.M. Jackson, D. Xu, J. Huang, R.J. Price, D.V. Volman, Earth, Moon, and Planets 89 (2002) 197.

(16) X. Dadong, H. Jianhua, W.M. Jackson, J. Chem. Phys. 120 (2004) 3051.

(17) G. Black, L.E. Jusinski, J. Chem. Phys. 82 (1985) 789.

(18) J. Zoval, D. Imre, P. Ashjian, V.A. Apkarian, Chem. Phys. Lett. 197 (1992) 549.

(19) H. Okabe: Photochemistry of Small Molecules, Wiley, New York, 1978.

(20) MultiWell-2008.2 Software, designed and maintained by John R. Barker with contributions from Nicholas F. Ortiz, Jack M. Preses, Lawrence L. Lohr, Andrea Maranzana, and Philip J. Stimac, University of Michigan, Ann Arbor, MI, http://aoss.engin.umich.edu/multiwell/, 2008.

(21) P.A. Denis, J. Sulfur Chem. 29 (2008) 327.

(22) H. Hippler, J. Troe, H.J. Wendelken, J. Chem. Phys. 78 (1983) 6709.

(23) M. Heymann, H. Hippler, D. Nahr, H.J. Plach, J. Troe, J. Phys. Chem. 92 (1988) 5507.

(24) C. Zhou, PhD Thesis, University of Sydney, 2009.

(25) B. Maiti, G.C. Schatz, G. Lendvay, J. Phys. Chem. A 108 (2004) 8772. 


\section{Tables}

Table 1: Rate constants calculated from Multiwell simulations for channels on the singlet surface.

\begin{tabular}{|c|c|c|c|c|}
\hline \multicolumn{2}{|c|}{ Reaction Rate Parameter a } & A & $\mathrm{n}$ & $E$ \\
\hline$\underset{b}{\mathrm{H}_{2} \mathrm{~S}+\mathrm{S}+\mathrm{M}=\mathrm{HSSI}}$ & $1(3 a)$ & $2.40 \times 10^{21}$ & -1.612 & 7 \\
\hline $\mathrm{H}_{2} \mathrm{~S}+\mathrm{S}=\mathrm{HSSH}$ & $(3 a)^{b}$ & $6.38 \times 10^{7}$ & 1.280 & -2 \\
\hline $\mathrm{H}_{2} \mathrm{~S}+\mathrm{S}=\mathrm{SH}+\mathrm{SH}$ & (3b) & $1.18 \times 10^{18}$ & -1.685 & 25 \\
\hline
\end{tabular}

${ }^{a}$ Units cm-s-mol-K-kJ

b Troe falloff parameters for (3a): $\alpha=0.5, \mathrm{~T}^{* * *}=\mathrm{T}^{*}=726$.

Table 2: Sensitivity to parameter variations of the Multiwell predictions of reaction rates on the singlet surface at 296K. The sensitivities are calculated as average values of $\Delta \ln ($ rate $) / \Delta\left(\mathrm{E}, \mathrm{kJ} \mathrm{mol}^{-1}\right)$ for variations in the crossing barrier (E $\mathrm{ESC}_{\mathrm{ISC}} \pm 3 \mathrm{~kJ} \mathrm{~mol}^{-1}$ ) and $\Delta \ln (\mathrm{rate}) / \ln (\mathrm{F})$ for multiplicative variations $\mathrm{F}$ $(x 1.5, \div 1.5)$ in the crossing efficiency ( $\eta$ ISC) and $<\Delta \mathrm{E}_{\text {down }}>$.

\begin{tabular}{l|cccc|}
\cline { 2 - 5 } & \multicolumn{2}{c}{$\mathbf{p = 0 . 0 1 3}$} & \multicolumn{2}{c|}{$\mathbf{p = 0 . 5 5}$} \\
& R. 3a & R. 3b & R. 3a & R. 3b \\
\hline$E_{\text {ISC }}$ & -0.37 & -0.08 & -0.40 & -0.06 \\
$\eta_{\text {ISC }}$ & 0.14 & 0.64 & 0.61 & 0.56 \\
$\left\langle\Delta E_{\text {down }}>\right.$ & 1.50 & 0.02 & 0.73 & 0.01 \\
\hline
\end{tabular}




\section{Figure Captions}

Figure 1. Potential energy surface for the reaction $\mathrm{H}_{2}+\mathrm{S}$ [9] with updated energy for $\mathrm{H}_{2} \mathrm{SS}[21]$.

Figure 2. Plot of pseudo-first-order rate constant for consumption of $\mathrm{S}\left({ }^{3} \mathrm{P}\right)$ by $\mathrm{H}_{2} \mathrm{~S}$ at $658 \mathrm{~K}$ in Ar bath gas at a total pressure of 29 mbar. Error bars represent $2 \sigma$. The inset shows the fluorescence decay corresponding to the filled symbol.

Figure 3: Rate constants for reaction (1) as a function of pressure. The bath gas is argon. The points show the experimental data while the lines are the predictions of the model (see Discussion).

Figure 4: Comparison of experimental and predicted bimolecular rate constants for the reaction of $\mathrm{H}_{2} \mathrm{~S}+\mathrm{S}$. The present data are shown for 0.013 bar $(\boldsymbol{\nabla})$ and 0.55 bar $(\mathbf{A})$; the data of Shiina [8] are shown as $(\boldsymbol{X})$ for the original data and as (O) after correction for secondary reactions (see text). The solid lines show the rates of the various processes obtained in the Multiwell simulations (Table 1): R2, R3 (a, p $\rightarrow \infty)$ and R3 $(b, p \rightarrow 0)$. The dashed lines show the overall rates from the Multiwell simulations at 0.013 and 0.55 bar. The dotted lines show the contributions of R3a and R3b - these are very close to the dashed lines and difficult to discern. 


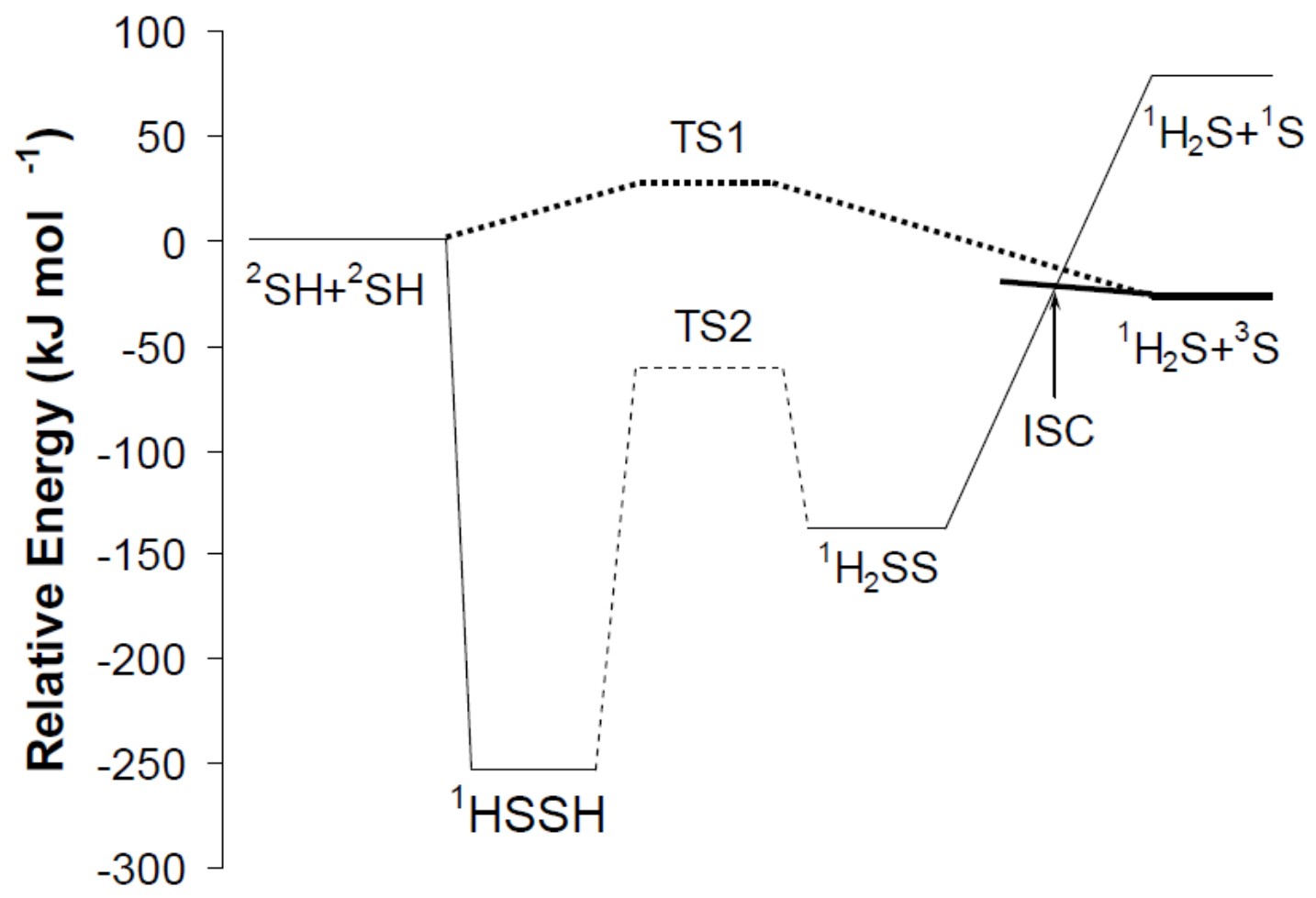

Figure 1. Potential energy surface for the reaction $\mathrm{H}_{2} \mathrm{~S}+\mathrm{S}$ [9] with updated energy for $\mathrm{H}_{2} \mathrm{SS}$ [21]. 


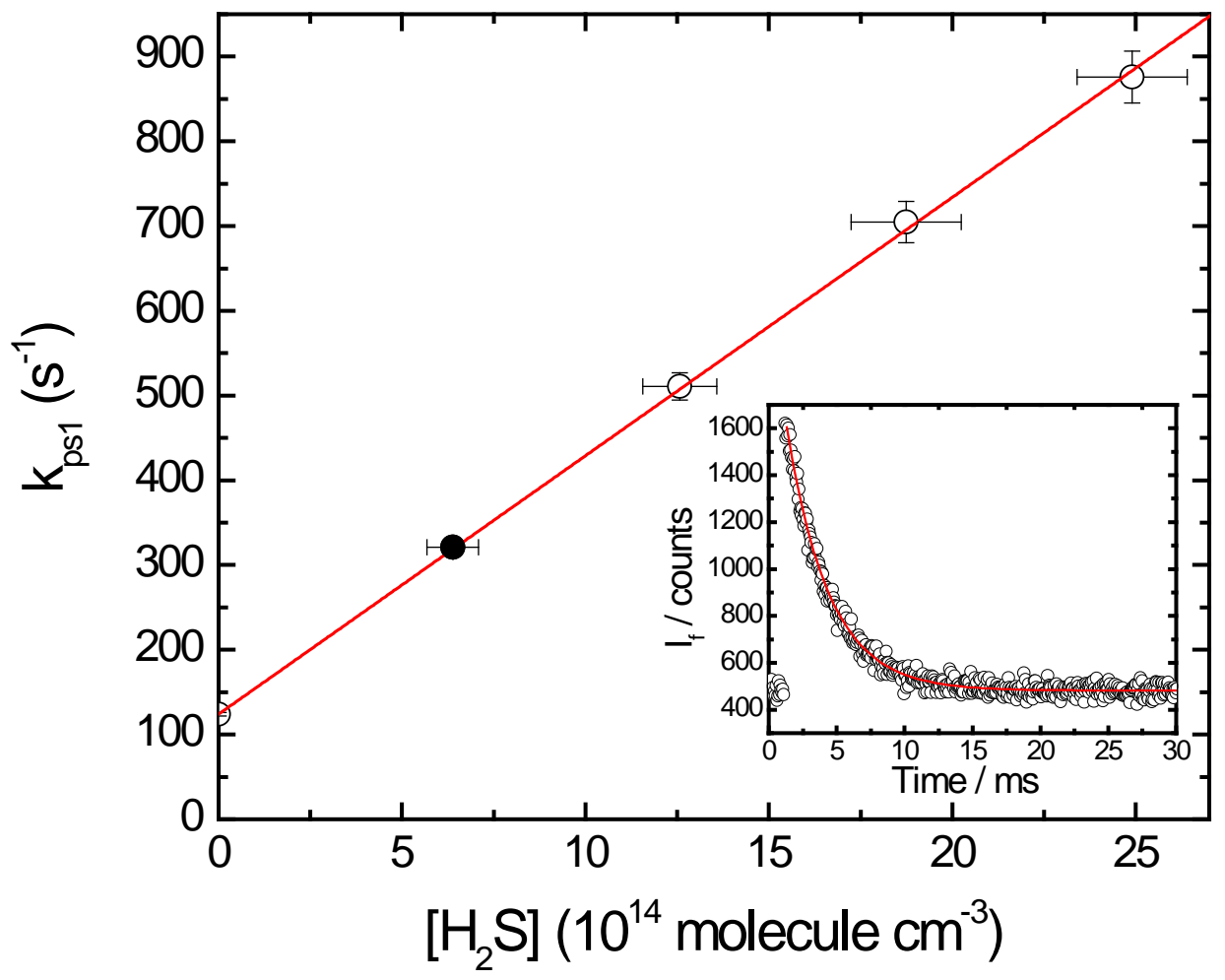

Figure 2. Plot of pseudo-first-order rate constant for consumption of $\mathrm{S}\left({ }^{3} \mathrm{P}\right)$ by $\mathrm{H}_{2} \mathrm{~S}$ at $658 \mathrm{~K}$ in Ar bath gas at a total pressure of 29 mbar. Error bars represent $2 \sigma$. The inset shows the fluorescence decay corresponding to the filled symbol. 


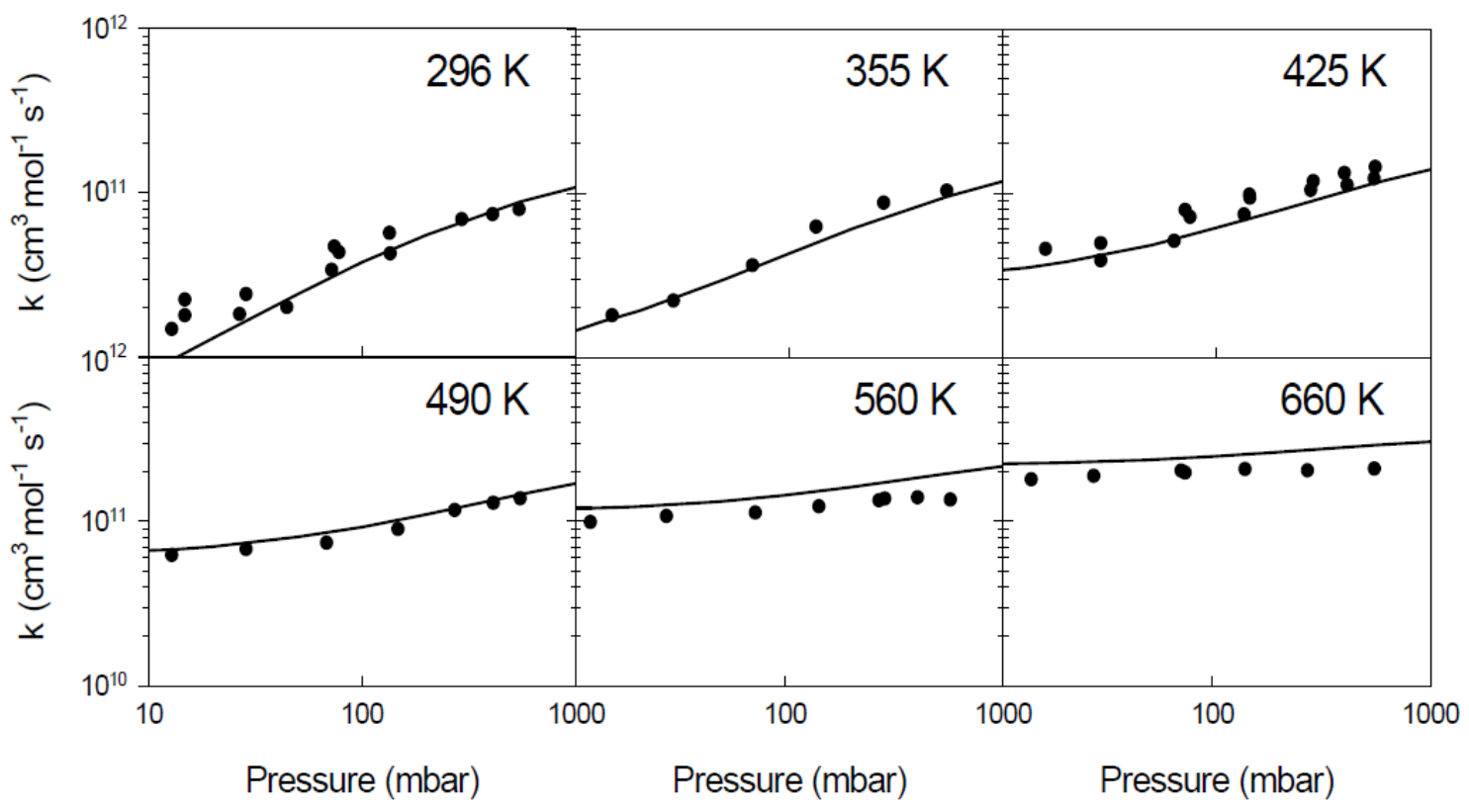

Figure 3: Rate constants for reaction (1) as a function of pressure. The bath gas is argon. The points show the experimental data while the lines are the predictions of the model (see Discussion). 


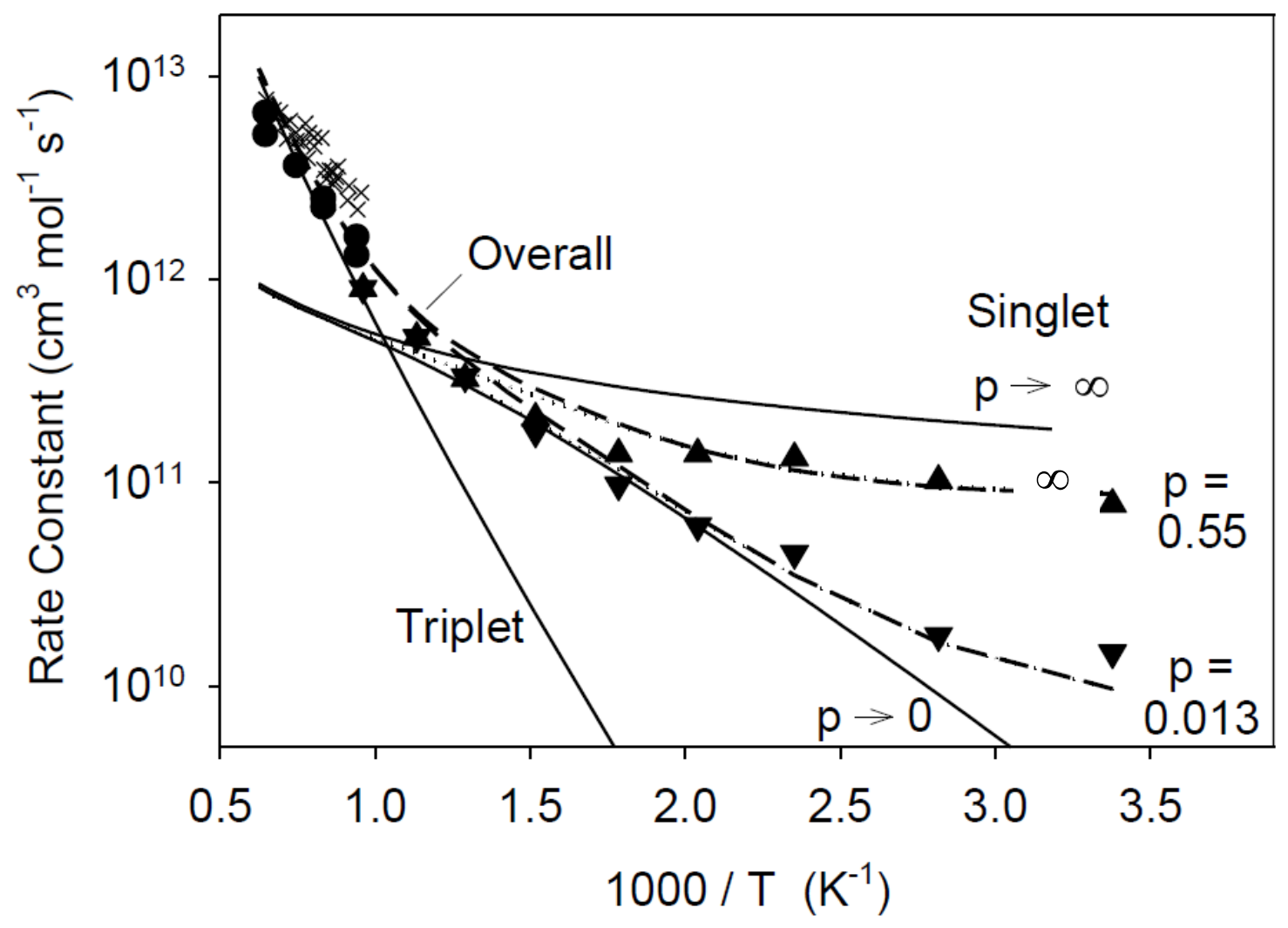

Figure 4: Comparison of experimental and predicted bimolecular rate constants for the reaction of $\mathrm{H}_{2} \mathrm{~S}+\mathrm{S}$. The present data are shown for 0.013 bar $(\boldsymbol{\nabla})$ and 0.55 bar ( $\mathbf{A})$; the data of Shiina [8] are shown as $(X)$ for the original data and as (O) after correction for secondary reactions (see text). The solid lines show the rates of the various processes obtained in the Multiwell simulations

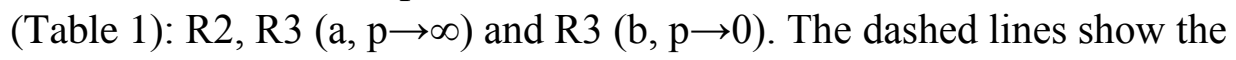
overall rates from the Multiwell simulations at 0.013 and 0.55 bar. The dotted lines show the contributions of R3a and R3b - these are very close to the dashed lines and difficult to discern. 
Figures at printing size
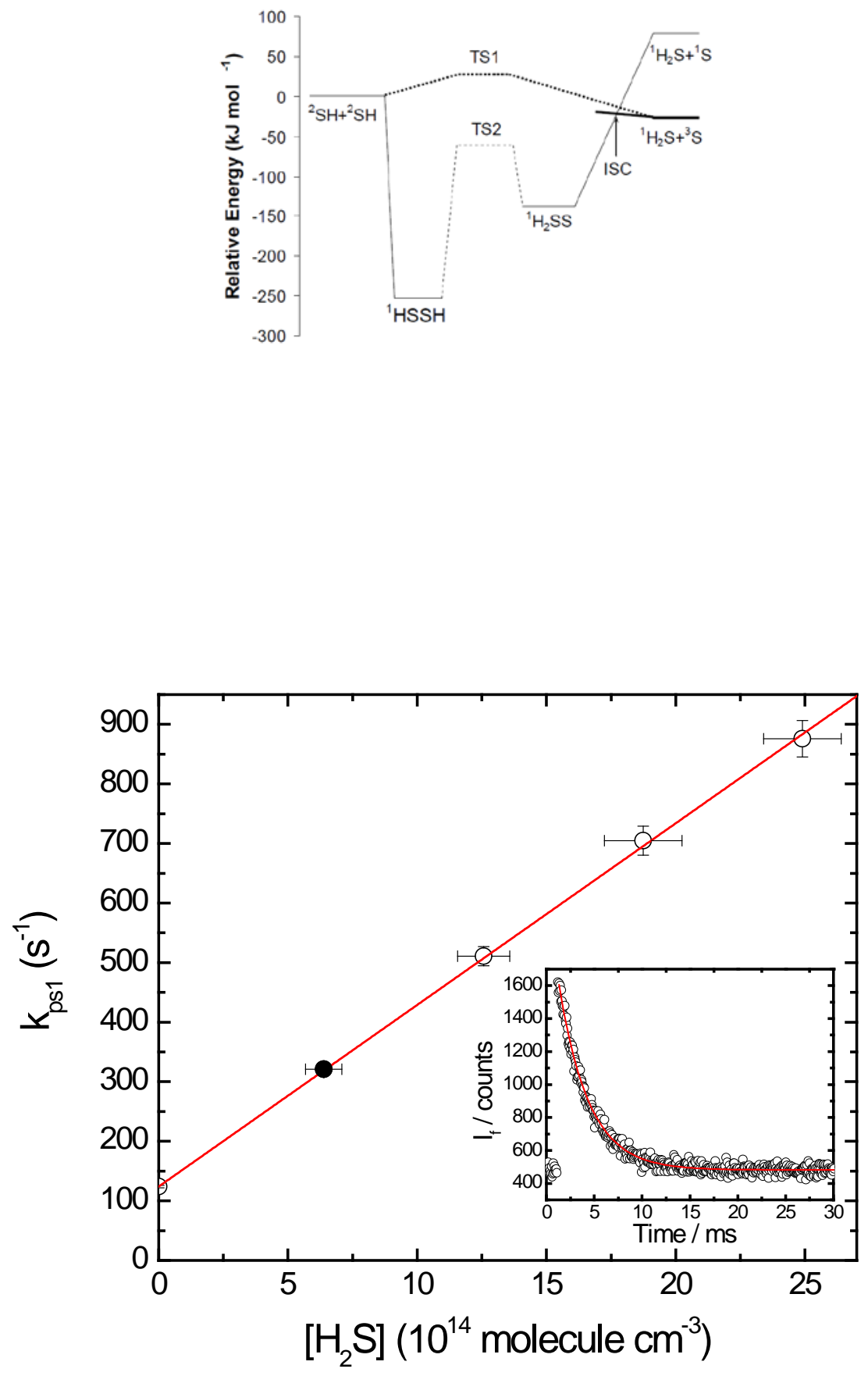

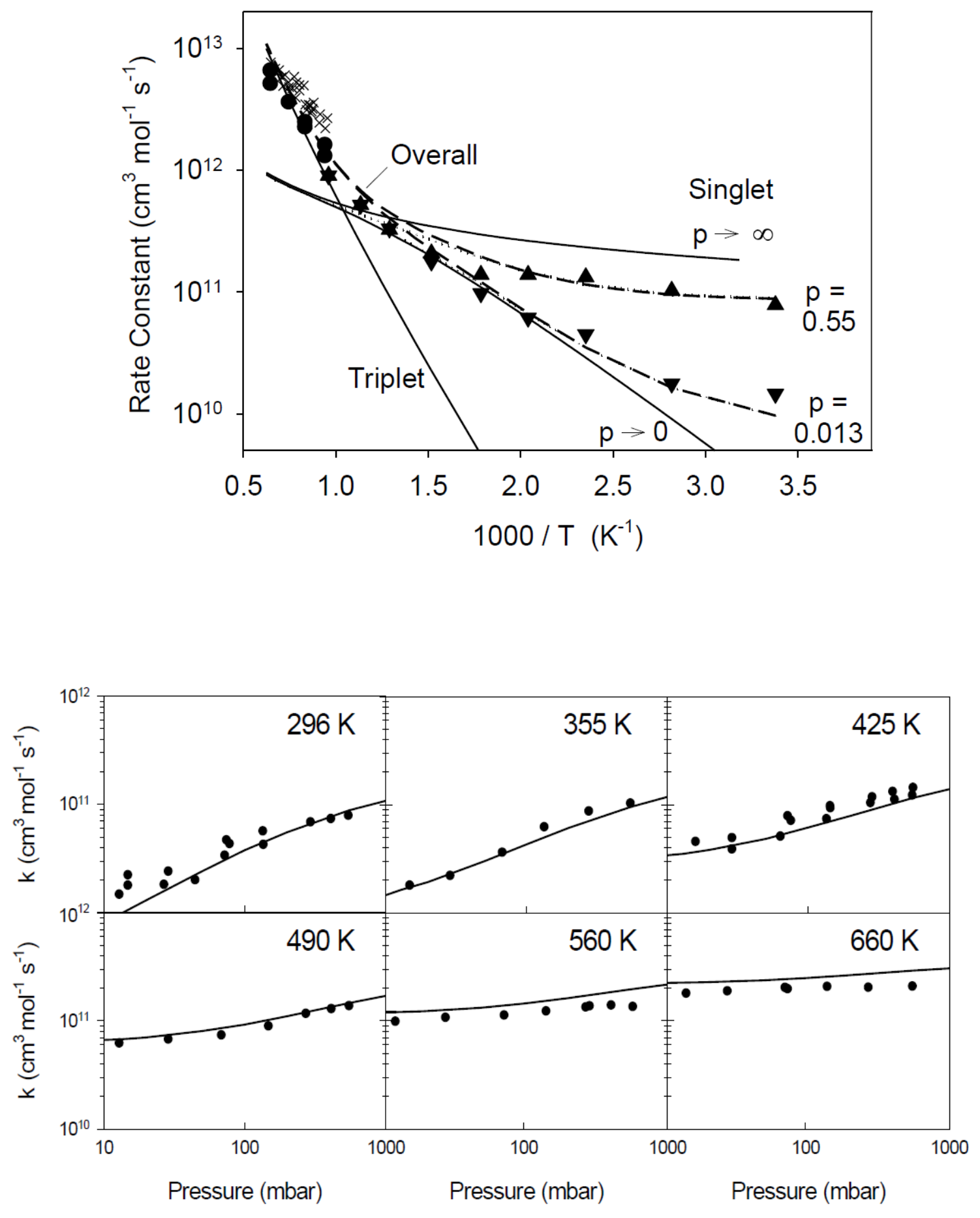